\title{
Le dialecte niçois : usage consacré et parler de la rue
}

\author{
Del Giudice, Philippe \\ Univ. Nice Sophia Antipolis, CNRS, BCL, UMR 7320 \\ phdelgiudice@gmail.com
}

\section{Introduction}

Quiconque s'engage - comme nous - dans une entreprise lexicographique d'envergure se trouve confronté à la question de la variation. Dans quelle mesure la prendre en compte ? Quelle place lui réserver dans l'outil et comment la présenter? De telles interrogations a priori banales sont essentielles lorsque l'objet d'étude est le dialecte occitan de Nice. Frontalier, à la fois très évolué et très archaïque, maritime mais en lien constant avec la montagne, il a toujours déchaîné les passions, donnant lieu à tous types de représentations.

Or, si la variation du niçois est désormais très bien documentée des points de vue diachronique et diatopique $^{1}$, cela ne suffit pas pour saisir la complexité de ce parler. Un regard sur les sociolectes de la langue régionale sera d'ailleurs l'occasion d'établir ici l'existence d'un décalage entre le niçois véhiculaire des passionnés de la langue et la pratique inapprivoisée des voies obscures du Vieux-Nice. Regorgeant d'exemples, ces quelques lignes ont l'espoir de mieux faire connaître le niçois tel qu'il est effectivement parlé, tout en posant deux questions : sa fantaisie n'était-elle pas digne de davantage d'attention? Et ne doit-il pas plus que jamais servir de garde-fou à une normalisation nécessairement artificielle?

\section{La situation sociolinguistique générale du niçois}

La question occitane est avant tout une affaire de diglossie (Lafont 1971, Gardy \& Lafont 1981). Or, contrairement à d'autres régions $d^{\prime}{ }^{2} c^{2}$, il n'existe pas pour le pays niçois d'étude sociolinguistique s'intéressant au dialecte. Difficile dans ces conditions d'évaluer avec une acuité parfaite la place qu'occupe le niçois dans son espace, sur le plan à la fois du développement de la pratique comme sur celui des représentations dont la langue fait l'objet. Quelques jalons aideront cependant à se faire une idée de la situation.

\subsection{Le réservoir de locuteurs}

Vouloir estimer au plus près le nombre de locuteurs reviendrait à abuser d'extrapolations et d'approximations. En appliquant la méthode de calcul de Bernissan (2012), il faudrait considérer que dans l'ensemble des Alpes Maritimes les locuteurs natifs (ou locuteurs primaires) ont disparu et que le nombre de néolocuteurs (ou locuteurs secondaires) s'élève à 1427 personnes. Si un tel chiffre, alarmant, donne un ordre d'idée, il ne faut pas oublier qu'il est obtenu au moyen d'une étude qui englobe une trentaine de départements et qui ne se propose donc pas de rendre compte de la situation particulière de l'espace niçois décrite infra (\$2.4). En 1984, en l'absence d'enquête rigoureuse, on estimait que la masse démographique de la zone recouverte par le niçois côtier comprenait «probablement moins de $10 \%$ de nissardophones » (soit 40.000 personnes au plus), qui avaient à l'époque dans leur majorité «plus de 50 ou 60 ans » (Gasiglia, 1984 : 72-73). Aujourd'hui l'on retrouve cette population de locuteurs primaires décimée et très avancée dans l'âge. Elle doit cependant être complétée par un nombre croissant de néolocuteurs. Existant de façon marginale au début des années 1940, l'enseignement du niçois n'a cessé de se développer en même temps que la transmission familiale disparaissait. Entré dans l'Éducation Nationale, présent de l'école maternelle à l'Université, il touche un public modeste mais en constante progression. Dans le primaire, la première école bilingue a ainsi été créée en 2012-2013. Selon le 
Rectorat, on comptait en 2013 dans les établissements secondaires des Alpes-Maritimes 1221 élèves d'occitan (à l'ouest du département le dialecte de référence n'est pas le niçois mais le provençal), répartis équitablement entre collèges et lycées. En outre, une cinquantaine d'étudiants apprend le niçois chaque année à l'Université. Plusieurs associations (l'Acadèmia nissarda, l'IEO, Nissart per tougiou, le Ccoc país nissart...) proposent également des cours de langue. Il est entendu qu'une partie des apprenants n'aura jamais une maîtrise courante du dialecte. D'ailleurs, en l'état actuel des choses, bien peu de néolocuteurs auront l'occasion d'y avoir recours dans leur vie quotidienne. Quoique l'on ne puisse s'appuyer que sur ces éléments d'information très vagues, il apparaît difficilement contestable qu'au regard du demi-million d'habitants de l'agglomération niçoise la communauté niçardophone ne représente qu'une proportion dérisoire. À côté de cela subsiste la population de non-locuteurs fortement imprégnés (enfants de locuteurs natifs ou ayant grandi dans des quartiers où l'occitan de Nice était massivement employé). Elle ne semble pas négligeable mais elle est non documentée. Les individus qui la composent sont généralement sensibles aux initiatives en faveur du dialecte.

\subsection{L'action pour le maintien du niçois}

Parallèlement au fort recul ces dernières années de la communication spontanée de personne à personne, caractéristique des locuteurs natifs, ont émergé des îlots de sauvegarde qui garantissent au dialecte un espace social. Sur ce point, la situation niçoise ne diffère en rien de l'ensemble de l'Occitanie. La salle de classe est notamment devenue un sanctuaire. Dans la transmission du savoir linguistique, le rôle de l'Éducation Nationale et de l'Université vient d'être évoqué, conjointement à celui des associations. Ces dernières profitent de l'engagement des collectivités locales. Depuis 2008, la Mairie de Nice dispose d'une délégation "patrimoine historique, archéologique, langue et culture niçoises". Cette délégation s'efforce de défendre les intérêts des acteurs culturels, propose des cours de langue aux personnels de la Mairie et développe l'emploi du dialecte lors d'événements culturels ou dans les documents et affiches émanant des services municipaux. À l'instar du Conseil Général des Alpes-Maritimes et du Conseil Régional de PACA, la ville apporte un soutien financier à la création et aux structures de maintenance.

Car le niçois arrive tout de même à sortir de l'enseignement. Les publications littéraires sont assez régulières, la revue (bilingue et bimestrielle) Lou Sourgentin, fondée en 1970, est tirée à 3000 exemplaires et la langue n'est pas totalement absente d'internet, où des forums, des blogs et certains sites l'emploient. Le grand quotidien de Nice ne laisse quant à lui au dialecte qu'une courte chronique hebdomadaire. D'autres médias permettent en revanche d'entendre le niçois. La seule émission télévisée en occitan de l'aire provençale, Vaqui, donne parfois la parole à des locuteurs niçois. Deux stations de la radio hertzienne (France Bleu Azur et Fréquence K) ouvrent leurs antennes à la langue régionale, chaque matin pour l'une, le vendredi soir pour l'autre, tandis que Ràdio Nissa Pantai diffuse en continu sur internet. La production musicale se porte elle aussi assez bien. Un public jeune se montre enthousiaste pour la chanson niçoise. Le grand succès des événements culturels et festifs (organisés par des associations) comme le Festivous ou le Festin d'aquì témoignent de cet engouement. Il ne faudrait pas oublier le théâtre dialectal, si populaire à Nice, qui compte plusieurs troupes, la plupart ayant plusieurs dizaines d'années.

Qu'elle soit représentée par des chanteurs, des auteurs, des blogueurs, des journalistes ou des membres d'associations, la maintenance est assez fortement liée au monde de l'éducation. Le Théâtre niçois de Francis Gag, fondé dans les années 1930, compte bien évidemment des enseignants d'occitan-langue d'oc parmi sa trentaine de membres bénévoles et a signé une convention en 2011 avec l'Académie de Nice, officialisant l'accueil de 400 élèves par spectacle. Lou Sourgentin est, lui, issu du monde enseignant auquel appartient une bonne part de ses auteurs. Les cours associatifs sont enfin souvent confiés à des personnes exerçant dans les établissements publics.

Comme ailleurs, la pérennité de l'occitan à Nice dépend donc avant tout des acteurs institutionnels d'une part et des initiatives individuelles ou associatives (le plus souvent bénévoles) de l'autre, le lien entre les deux univers étant assuré en grande partie par des enseignants, devenus les véritables dépositaires de la langue. 


\subsection{La grille de lecture occitane}

S'agissant des représentations, la confrontation des données du niçois avec le résultat des recherches sur l'ensemble occitan est instructive. Le cadre théorique proposé par la sociolinguistique occitane part, indirectement, de l'interprétation anglo-saxonne de la diglossie : une variété haute, prestigieuse, la langue A (pour nous le français) est superposée à une langue historique, la langue $\mathrm{B}$ (ici l'occitan) faisant automatiquement de cette dernière la variété basse de la diglossie. L'école catalane, tout en admettant dans une certaine mesure ce rapport Haut $v s$ Bas, fait remarquer qu'un modèle qui croit au «maintien stable plutôt qu'au remplacement d'un code par l'autre au cours du temps ${ }^{3} »$ (J. Fishman 1967 : 29-30) ainsi que sur le caractère "non-conflictuel» de ces codes n'est pas à même d'expliquer la réduction constante, au fil du temps, de l'espace social de la variété dominée au profit de la langue prestigieuse. Au contraire, Aracil (1965) et Ninyoles (1969) prouvent que la diglossie n'est pas figée mais constitue une dynamique mue par le conflit linguistique. Kremnitz (1980:24) résume les aboutissements possibles de la diglossie selon cette vision des choses : « ou bien la normalisation de la langue dominée [...] ou bien [sa] substitution définitive par [la langue] A ». Ferguson (1959) avait déjà observé qu'une spécialisation de fonction instaurée entre les deux codes limitait le champ d'usage de la langue basse à l'échange familier ou informel, réservant la grande culture et l'usage officiel à la langue dominante. Gardy \& Lafont (1981) développent la notion de fonctionnement diglossique (devenue depuis distribution fonctionnelle, cf. Lamuela 1994) et ses conséquences : le conflit, réactivé à chaque prise de parole, est invisible en synchronie puisqu'il se concrétise dans un partage. Non exprimé en tant que tel, il n'est pas observable et ne se prête pas à l'étude à moins que l'on suive son évolution dans le temps. C'est-à-dire que chaque cas de diglossie ne peut être analysé que dans sa dimension historique. Pour Gardy \& Lafont (1981:77) «par delà les manifestations synchroniques de la diglossie, ce sont donc les mécanismes de son histoire, au niveau des pratiques, certes, mais également au niveau de l'élaboration des notions qui composent l'idéologie diglossique, qu'il convient de dégager et d'analyser ».

L'un des principaux apports des continuateurs occitans de l'école catalane se situe justement dans la mise au clair des étapes historiques du processus diglossique qui conduit au remplacement progressif de la langue d'oc par la langue nationale. Il faut retenir de ce processus qu'il est long et insidieux (monopôle du français dans l'écrit dès le XVIème siècle renforcé par l'Ordonnance de Villers-Cotterêts de 1539, spécialisation fonctionnelle avancée au XVIIème, conversion de l'élite et début de l'accès au français pour toutes les classes au XVIIIème, mise en place dans les siècles suivants d'un appareil d'état en vue de l'unification linguistique de la France ${ }^{4}$ ), qu'il est rapidement intégré par la population (en 1615, déjà, le jurisconsulte provençal Jules Raymond écrit dans ses Antiquités de la ville de Marseille ${ }^{5}$ : «la Provence a été unie au royaume de France, on a commencé d'y parler à demi françois, et sans doute qu'en peu de temps, on n'y parlera que françois ») et qu'il trouve sa résolution au moyen de l'étape ultime du patois, « un concept dont la fonction est bien de caractériser la diglossie, plus : de la dénoncer » (au détriment de la langue B). « Il sanctionne la situation de non-pouvoir dans laquelle se trouve la langue dominée (...); cette dépossession s'accompagne d'une extrême différenciation territoriale, de telle sorte que la langue dominée, pour ainsi dire invertébrée, n'a plus de position géographique, mais une simple position socioculturelle » (Gardy \& Lafont $1981: 83$ ).

\subsection{La spécificité niçoise}

Le cadre général occitan ne suffit pas à se représenter le niveau de complexité du cas niçois. Évidemment, le niçois est à l'heure actuelle écrasé sous le poids du français. Toutefois, la production historique de la diglossie a eu ses modalités propres à Nice, donnant lieu à d'autres représentations que celles du modèle habituel ${ }^{6}$. Alors que, partout dans le sud de la France, l'inexorable effacement de l'occitan face au français a été de bonne heure annoncé et longuement mûri, le language switch fut à Nice d'une grande brutalité. Déjà, à propos de la période d'implantation du français, Kremnitz (2002a : 20) évoque un « décalage temporel » des « territoires appartenant à la Savoie autour de Nice ». Ce décalage est jugé léger. Il semble surtout que dans le processus diglossique cette implantation soit moins prégnante ${ }^{7}$. On s'aperçoit notamment que le sentiment de menace arrive fort tardivement dans le Comté. Le catastrophisme qui a gagné la Provence dans la première moitié du XIXème siècle ne se retrouve pas à Nice. En 1840, Victor 
Gelu ${ }^{8}$ écrit: «l'idiome provençal se meurt. [...] Avant trente ans, cette langue sera aussi difficile à expliquer que la langue des hiéroglyphes pour les quatre-vingt-dix-neuf centièmes de notre population marseillaise ». C'est tout le contraire que l'on trouve chez l'auteur niçois J.-R. Rancher qui juge en 1830 qu' « à partir de [s]es écrits, il sera facile de dèterminer dans la suite des siècles les révolutions qu'aurà éprouvé une langue qui n'a pour base que le caprice de ceux qui la parlent ${ }^{9} \gg$ ou chez l'abbé Micèu qui adresse explicitement en 1840 sa Grammatica Nissarda (rédigée intégralement en occitan) aussi bien aux Niçois qu'aux riches hivernants et aux employés administratifs étrangers. Ce sentiment de pérennité de la langue locale et d'adaptabilité des étrangers ne se démentira jamais. C'est à croire que la communauté des niçardophones n'a pas vu les choses arriver. Aujourd'hui, les personnes élevées dans des quartiers niçois (souvent des non-locuteurs fortement imprégnés) semblent très étonnées que la population ne parle plus le dialecte.

La conversion linguistique fut d'autant plus spectaculaire que le niçois, au contraire du provençal ou du languedocien, semble ne jamais connaître la désaffection des classes supérieures et dirigeantes. Partant, il n'est pas associé à une couche sociale en particulier. Selon (Kremnitz 2002a : 26-27), à la Révolution « l'emploi du français ou de l'occitan varie selon les couches sociales ». Or, à l'image de Pierre Devoluy, cité par Gasiglia (1984 : 74), les Provençaux s'étonnent qu' «alors qu'à Aix, à Avignon, à Montpellier, la langue d'Oc est trop souvent tenue à mépris par les "Messieurs" (li moussurot), ce sont, au contraire, à Nice, comme autrefois dans toute la Provence et le Languedoc, les meilleures familles du pays qui se font honneur de parler leur idiome national ». De nos jours il reste encore des avocats et des médecins qui parlent niçois, parfois dans leur cabinet. Les Maires de Nice et leurs adjoints dialectophones n'ont jamais répugné à l'employer en public. C'est une tradition qui n'est pas totalement perdue. L'équipe municipale ne s'est d'ailleurs mise au français que bien tardivement : «dans les réunions [du Conseil Municipal] les affaires se traitent en notre dialecte quoique les délibérations soient rédigées en langue française » écrit Toselli (1878 : XV). La distribution fonctionnelle est donc à relativiser dans l'espace niçois. En 2014, les locuteurs, y compris les locuteurs primaires des couches sociales inférieures, utilisent sans se poser de question la langue régionale pour débattre de politique ou d'économie. Le processus diglossique est donc structurellement "en retard" à Nice par rapport aux autres régions occitanes.

Notamment, porté par la fierté identitaire très prononcée des autochtones que l'on doit aux avatars de l'histoire, le dialecte est tenu en bonne estime par les locaux et sa légitimité sur le sol naturel perçue comme une évidence. Il est relativement difficile de trouver trace des complexes ou de la honte qui lui sont associés dans d'autres régions méridionales, au point qu'il est nécessaire de s'interroger sur la pertinence du concept de patois. Le mot lui-même, qui pénètre, en français et en dialecte, dans les publications niçoises au début du XIXème siècle à côté de nissart semble avoir été définitivement chassé par ce dernier avant le tournant du XXème siècle. Dans l'esprit des locuteurs, instruits ou non, il ne semble pas intégré. La communauté linguistique l'a refusé.

Jouant à la fois comme cause et comme conséquence du système de représentation, un fait décisif est que le niçois est le seul exemple vivant d'occitan urbain ${ }^{10}$. Si pour Bernissan $(2012: 491)$ «on sait que l'occitan a depuis longtemps déserté les centres urbains (circa 1900) », faisant un peu partout de la langue d'oc l'idiome de la paysannerie et du temps passé, le niçois est plutôt vu dans son espace comme le parler référentiel, celui d'une grande ville au fort rayonnement local. À l'époque même où il est menacé, il reste conquérant dans les tranches d'âge qui le pratiquent. Au XXème siècle on lui découvre un fort pouvoir d'extension géographique. D'une part, tout montre qu'il pénètre l'arrière-pays et se diffuse le long des côtes en se substituant progressivement aux variétés locales ${ }^{11}$. D'autre part, il est souvent connu des dialectophones de l'ancien Comté de Nice, y compris dans des communes où la variété locale a résisté à l'invasion du parler urbain ${ }^{12}$. Nous avons constaté ce phénomène jusque dans la vallée de la Roya, pourtant non occitanophone (zone du ligurien, cf. Dalbera 1989 et Forner 2001). Cette force d'attraction est confirmée par la capacité d'intégration des populations immigrées. En particulier, les populations provenant des territoires de l'actuelle Italie se sont toujours mises rapidement au niçois, produisant des individus capables de parler aussi bien le dialecte de leur région d'origine que celui de leur ville d'accueil. Si le cas des Piémontais est connu (Gasiglia 1984 : 18), nous nous sommes intéressés aux descendants d'immigrés de Senerchia (Provincia d'Avellino). Nos enregistrements témoignent de certains 
cas de trilinguisme senerchiais/niçois/français, toutes langues parlées parfaitement. Nous avons également rencontré des personnes de tous horizons, y compris des Français, arrivés entre 1950 et 1970 et capables de s'exprimer dans un occitan de base, appris sur leur lieu de travail au contact des Niçois.

On comprend mieux que le niçois soit perçu par ses locuteurs comme une langue parmi d'autres. C'est que jusqu'à une époque très récente la diglossie ne peut se réduire au schéma habituel patois vs français. Longtemps, le niçois coexiste dans son propre espace avec l'italien (langue officielle de 1561 à 1860), le français, le piémontais (certains journaux humoristiques proposent des articles écrits dans le niçois piémontisant des immigrés Nord-Italiens), le ligurien, les autres dialectes d'Italie, l'anglais et le russe des hivernants, les variétés occitanes très différenciées du Comté et le provençal maritime, généralement considéré, à tort, comme une toute autre langue.

Au vu de sa position sociolinguistique plutôt avantageuse pour un dialecte de France, de sa vigueur et de son pouvoir d'intégration admirable, on peut donc se demander si le coup d'arrêt brutal (une conversion linguistique aussi rapide qu'imprévue) ne doit pas aussi être interprété en partie comme une submersion démographique ${ }^{13}$ dans une France du XXème siècle par ailleurs marquée par les guerres et présentant une forte union nationale.

Demeure une particularité. La question centrale, qui cristallise bon nombre des problèmes de la représentation, c'est celle de la "nationalité" du dialecte de Nice. Enjeu politique souvent débattu entre Français et Italiens (Gasiglia 1984 : 83-91), le sujet trouve différentes réponses au sein de la population. Une interprétation particulariste fait $\mathrm{du}$ niçois une langue originale et parfaitement autonome. Pour d'aucuns il est le résultat d'un mélange de langues, vivantes et anciennes. Pour d'autres il a des affinités avec le piémontais, le ligurien voire même le sarde (influence de l'histoire politique), quand il n'est pas tout simplement lui-même un italien dialectal... L'occitanité du niçois est défendue par un certain nombre d'auteurs des XIXème et XXème siècles, mais le monde littéraire est loin d'être imperméable aux filiations fantasmées ${ }^{14}$. Au XXème siècle, en dehors des personnes ayant suivi des cours, les locuteurs ne sont guère informés du fait occitan. C'est dans un tel contexte sociolinguistique qu'il faut aborder la question des usages de référence qui semblent émerger.

\subsection{Quel avenir pour le niçois : les conditions d'un maintien}

En dépit du sentiment identitaire prononcé d'une partie de la jeunesse niçoise, la question de l'avenir du dialecte se pose. Les conditions actuelles de sa survie sont précaires. Une reconquête de l'espace social est-elle envisageable? Si l'on accepte l'idée que la tendance ne peut être inversée que grâce à la normalisation, il faut voir où en sont les Niçois dans ce chantier. Ce processus comporte deux volets complémentaires. Sur le plan institutionnel c'est la planification linguistique. Sur le plan linguistique c'est la normativisation, autrement dit l'élaboration d'une langue standard.

À l'échelle locale, malgré un phénomène de convergence croissante des collectivités, du Rectorat et des acteurs culturels, il n'existe pas encore de plan concerté de revitalisation sociale de la langue à moyen et long terme. Des efforts méritoires portent à bout de bras une certaine forme de subsistance de l'occitan, mais personne ne s'interroge sur le rôle qu'il peut ou doit jouer dans la société. Impossible dans ces conditions de fixer des objectifs clairs et, a fortiori, d'évaluer les résultats. Sauf revirement majeur, on se dirige vers une conservation mémorielle de type mythique ayant valeur de symbole identitaire. Dans un contexte plus global, les Niçois se réjouissent des avancées obtenues par les associations panoccitanes (notamment en termes d'enseignement et de statut des langues régionales) mais, au sein même des institutions, des opposants farouches à toute forme d'occitanisme demeurent. On est loin d'une véritable planification.

Concernant le volet normatif, il faut signaler la préexistence, à un premier niveau, d'une norme tacite du niçois, qui sert de base à la langue héritée actuelle. Née dans le peuple au cours d'un processus naturel, elle est intéressante en ce qui concerne les mots d'évolution qui ont subi toutes les étapes du changement phonétique à partir du latin vulgaire. On constate qu'elle naît d'un sentiment particulariste. Il semble, selon Dalbera (1988-1989), qu'à une époque guère éloignée (vers la fin du XVIIIème siècle ?) « un 
certain nombre d'évolutions, ramifiées en faisceau, aient pu produire un changement linguistique sensible et conduire à une nouvelle norme objective [...] ratifiant en quelque sorte a posteriori une évolution divergente avec le provençal» (on pourrait dire avec un occitan référentiel, quel qu'il soit). Cette norme objective demeure par nature assez souple, car soumise à une certaine variation. Il s'agit de savoir, à un second niveau, si le niçois a connu une normativisation. C'est-à-dire si un standard massivement accepté par la communauté linguistique a :

- effectué un traitement raisonné, systématique et explicite de la variation qui demeure dans l'usage des mots d'évolution tels qu'ils sont recueillis.

- Décidé d'un usage de référence dans des domaines où les solutions ne s'imposent pas d'ellesmêmes et dépendent grandement des options de nationalité (selon la problématique vue supra §2.4) : graphie, mots savants, critères d'acceptation (ou de rejet) des emprunts.

Clairement, un telle norme prescriptive n'existe pas, malgré l'émergence d'une pratique consacrée, issue de sources composites, qui soulève le problème du rapport entre la langue des locuteurs primaires et secondaires.

\section{La variation d'obédience et les sociolectes de norme}

Une observation minutieuse du niçois dans la diversité de ses manifestations conduit à identifier un type de variation peu évoqué. A priori inconnu du français, il apparaît dans les langues qui, ignorant leur nature et soumises à des pressions contradictoires, se retrouvent sous l'influence de plusieurs standards. Dans un tel cas, différentes catégories d'une même population suivront des normes opposées en fonction, entre autres choses, de leurs origines, de leur éducation, de leurs intérêts ou de la représentation qu'elles se font de leur idiome.

Le niçois contemporain ${ }^{15}$ a toujours été soumis à cette variation. Aujourd'hui encore, plusieurs poches de populations sont susceptibles d'être identifiées. Les voici, détachées en deux groupes principaux, d'une part le groupe "naturel" des locuteurs de la rue et d'autre part le groupe "académique" des mainteneurs (c'est-à-dire les personnes ou les groupements impliqués dans la défense et la transmission du dialecte que nous avons identifiés supra $\S 2.2$ : revues, associations, auteurs, enseignants, acteurs de théâtre, etc.).

\subsection{Le groupe "naturel"}

Le niçois de la rue, tel que nous le connaissons par notre contact avec les septuagénaires et octogénaires du Vieux-Nice, est employé de manière naturelle, spontanément et couramment, par les derniers locuteurs natifs. Fidèlement représenté, jusqu'à la seconde guerre mondiale, dans la presse et la littérature populaires, il a de nos jours complètement disparu de l'écrit. Peut-être est-il seulement mal connu des mainteneurs. Rémy Gasiglia s'est efforcé d'en rappeler certains traits et d'en défendre la valeur ${ }^{16}$. Dans ces pages, nous verserons à notre tour d'autres pièces, moins connues et parfois plus "sauvages", à ce dossier.

On pourrait définir l'usage auquel nous emprunterons tous nos exemples comme suit: XXème siècle, vieille ville, populaire, oral (ou oralisant), commun. Le XIXème siècle n'a pas été pris en compte car si la stabilité du lexique populaire est remarquable à toutes les époques, l'usage était alors tiraillé entre les emprunts savants au français et à l'italien. Cette incertitude dans la langue de la rue a progressivement disparu sous le poids écrasant du français.

\subsection{Le groupe "académique"}

Les courants académiques ont cette particularité de manier le niçois de telle sorte qu'il ait une apparence conforme aux positions défendues par leur école. À une époque où l'on écrit à peu près comme on parle et où la question de la nature de la langue régionale n'est pas encore réglée, c'est le groupe félibréen de l'Escola de Bellanda qui, le premier, voudra épurer le niçois pour lui rendre son caractère proprement occitan $^{17}$. Les excellents textes de la revue Lou Cairè ${ }^{18}$ témoignent de la réussite de l'entreprise. La 
langue, tout en s'appuyant sur une base populaire incontestablement niçoise, se nourrit du prestigieux modèle mistralien ${ }^{19}$. Parallèlement, un courant particulariste s'affaire, représenté dans toute sa splendeur par les publications d'Eugène Ghis et de Pierre Isnard ${ }^{20}$.

Après la Libération, la transmission familiale du dialecte recule dramatiquement. Les futurs mainteneurs devront étudier le niçois pour pouvoir l'employer, ce qui pose la question de leur niveau ${ }^{21}$. Dans ce contexte, davantage que l'usage courant, les ouvrages traitant de la langue deviennent au fil des ans la base commune de la connaissance du dialecte. Actuellement, tout l'académisme sans exception fonctionne sur ce socle partagé, légèrement retouché en fonction des préoccupations linguistiques des uns et des autres.

Il conviendrait cependant de partager ce groupe en deux branches, un courant principal qui ne donne lieu pour le moment à aucune remarque particulière et le courant occitaniste, soucieux de suivre au plus près les préconisations du mouvement unificateur de sensibilité alibertine. Comme cela fut le cas avec les premiers félibres locaux, mais de manière plus prononcée encore, la volonté de pureté occitane s'accompagne d'une certaine "centralisation" du niçois. Des lexèmes et des tournures tels que es pas grèu, una maion de li bèli, lo sègle XVII, particular, contemporanèu, seten, menimós ${ }^{22}$ se développent. Ne pouvant s'appuyer sur un standard régional clairement défini ${ }^{23}$, la démarche soulève à l'occasion des problèmes d'ordre morphologique ou phonétique. Un suffixe improductif comme - aís ${ }^{24}$, attesté à Nice soit dans des adjectifs soit dans des noms féminins (il devient alors -aíssa), reprend du service, sous une forme où la consonne intervocalique amuïe est rétablie, par exemple dans le nom (masculin) mescladís ('mélange' pour mesclum). Une importation telle que manlevar ('emprunter' pour empruntar, si faire prestar, pilhar en prèst) est du même acabit. Elle constitue un bon exemple de construction lexicale complément + verbe, assez courante dans le Languedoc (caplevar 'basculer', sanglaçar 'glacer le sang') mais en réalité peu représentative du parler de Nice. Dans le même genre, il faudrait se demander si la phonologie autorise les unités europèa, contemporanèa dont on attendrait qu'elles deviennent europea, contemporanea.

On pourrait schématiser les pratiques actuelles du niçois comme suit :

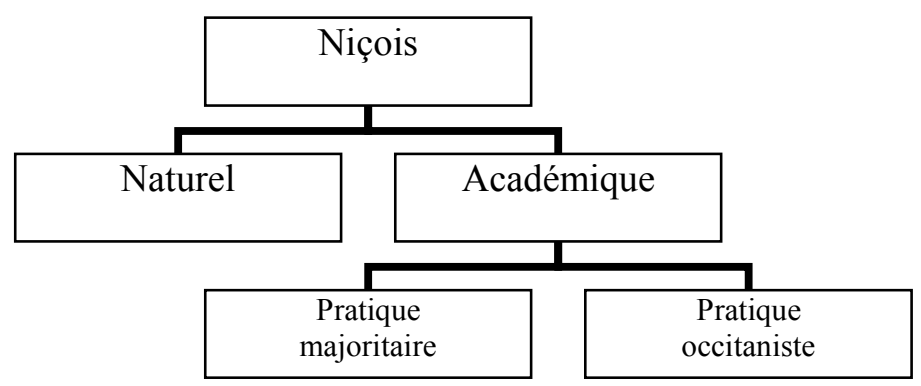

Ces divisions étant clarifiées, tâchons d'illustrer les différences entre le niçois naturel et le niçois académique pris dans son ensemble. L'idée est ici de mettre en lumière d'abord la langue des natifs puis d'interroger les pratiques normatives, sans jamais perdre de vue la comparaison entre les deux groupes.

\section{Le caractère bien trempé du niçois populaire}

\subsection{Des réalisations originales}

Un premier écart observable à l'oral entre le parler populaire et celui des milieux académiques concerne le comportement des voyelles. Cet écart peut, le cas échéant, refléter de légères divergences d'ordre phonologique. En voici quelques exemples ${ }^{25}$.

La perte, dans un nombre croissant de contextes, de l'opposition /e/ / / / a déjà été signalée ${ }^{26}$. Cette distinction est malgré tout encore présente chez la plupart des locuteurs du Vieux-Nice (zone pourtant 
réputée innovatrice). Ainsi, la voyelle tonique réalisée dans boulega 'bouge', messa 'messe', letra 'lettre', aquela 'celle-là' et frema 'femme' n'est pas la même que celle de pèça 'pièce', bella 'belle', prèga 'prie'ou tèsta 'tête'. Ainsi [s'et] signifiera 'soif' et [s'ct] 'sept'. Le milieu académique, guère aidé par l'accentuation graphique aléatoire des outils didactiques, est prompt à réaliser toutes ces voyelles $[\varepsilon]$.

Non phonologique, l'écart d'aperture de certaines voyelles finales accentuées est également intéressant. Un mot tel que acò s'achève par une voyelle ouverte ${ }^{27}$. La série voulentié, premié, quartié varie quant à elle entre des réalisations fermées [je], et ouvertes [je]. Les mainteneurs ne connaissent que la variante fermée dans cette position ${ }^{28}$.

En position prétonique, le nombre de $[\mathrm{u}]$ qui passent librement à [y] est remarquable. Coumun [kum' 'y $]$ 'W.C.', couhina [ku'ina] 'cuisine', pougne [p'une] 'poing', mousclà [muskl'a] 'hameçon', toujou [tudz' u] 'toujours' passent aisément à [kym'y'], [ky'ina], [p'yne], [myskl'a], [tydz' u]. Chez les locuteurs les moins instruits ce [y] est en outre relâché, se rapprochant du [Y] qu'il est possible d'entendre en Provence maritime ou dans le Languedoc oriental. Le groupe [ $\left.\urcorner^{\mathrm{n}}\right]$, pour sa part, disparait bien souvent au

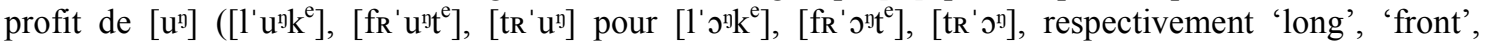
'tonnerre').

Le cas des diphtongues est particulièrement frappant. À côté de / $/ \mathrm{w} /$, nos aînés connaissent /ew/. Ce dernier apparaît dans des mots tels que eu 'lui', aqueu 'celui-là', beu 'il boit', veu 'je vois' que les mainteneurs réalisent systématiquement comme [' $\varepsilon w]$, [ak' $\varepsilon w],\left[b^{\prime} \varepsilon w^{29}\right],\left[v^{\prime} \varepsilon w\right]$. Que ce /ew/ soit également menacé dans la langue "du peuple" n'est pas ici remis en cause. Effectivement, il peut donner lieu à des stratégies d'évitement mais elles sont différentes de celle (l'ouverture) suivie par le monde académique. Dans la rue, beu, veu et aqueu se rapprochent plutôt, respectivement, de [b'yw], [vj'ew], [ak'yw] ou [akj'ew] / [ak'iw].

Ce sont de toute façon les diphtongues en général qui sont précaires. La prononciation très ouverte des mainteneurs les sauve. Le parler de la rue les massacre. Si le cas de [чє] devenu [œ] est connu ${ }^{30}$, les diphtongues descendantes sont tout aussi concernées. /ew/ peut être clairement, selon qu'il est tonique ou pas, réalisé [ø] ([døg'ut] 'dû' pour deugut) ou [œ] (Jeuse 'Jésus' prononcé [dz'œze]) tandis que [๖w] se réduit à [0] (gavòudola 'toupie'devenu [gav'odula]), parfois presque à [o] ( cò $^{31}$ pouvant s'approcher de $\left.\left[\mathrm{k}^{\prime} \mathrm{o}(\mathrm{w})\right]\right)$. Ce dernier cas tranche avec le [ow] académique, parfois réalisé presque comme [aw]. Quelques publications anciennes rendent compte de la prononciation non érudite, par exemple Fenouille \& Rondelly (1919: 72 et 87) avec Pouante-Nò et desenô (les normes graphiques actuelles notent bien entendu la diphtongue, par exemple : Pouònt-Nòu et dès-e-nòu). Ce qu'il faut retenir de ces formes, qui n'ont certes pas toutes vocation à devenir référentielles, c'est que certaines témoignent du maintien d'oppositions phonologiques. /'e/, /'ew/ et /'aw/ semblent bien distincts de /'E/, /'Ew/.et /'ow/ respectivement. En toute logique, l'orthoépie gagnerait sans doute à s'aligner sur la pratique héritée. Aucun ouvrage didactique n'en rend compte précisément.

Rien n'a été dit des consonnes. Elles sont également remarquables, notamment en position finale. En ville, outre la fréquence importante à laquelle les -t du participe passé sont omis ${ }^{32}$, la quantité de [s] finaux qui chutent est notable. Il n'est pas rare de trouver des locuteurs pour qui crous 'croix', brès 'berceau', bras 'bra', près 'prix', tros 'morceau' se limitent dans leur réalisation phonétique à [kR'u], [bR' $\varepsilon$ ], [bR'a], [pR' $\varepsilon]$, [tR'o]. Lorsqu'il n'y a pas ambiguïté, les formes verbales elles-mêmes peuvent être affectées, faisant écho au marseillais. Un de nos informateurs disait sans problème tu que la counouisse ${ }^{33}$.

\subsection{Une réserve lexicale}

Le vocabulaire des locuteurs du Vieux-Nice est surprenant. L'échange oral avec nos contemporains ou la lecture, par exemple, des journaux satiriques des années 1930 est toujours l'occasion de découvrir un fonds trop négligé par la lexicographie et donc difficilement accessible pour les mainteneurs.

L'absence du vocabulaire trivial ou de l'anatomie intime dans nos chastes dictionnaires était prévisible. Elle ampute néanmoins la langue d'un registre entier et de mots forts courants comme bouhà 'coïter', cucà (de même sens), bric 'maison close' ou fava (dans l'acception 'clitoris' ou 'gland du pénis'). Les locuteurs 
naturels ne sont pourtant pas limités dans ce domaine. Les lexies les plus osées existent, sont diverses et colorées.

On explique mal en revanche ce qui a pu reléguer au purgatoire une infinité de lexies telles que lou chapanas (seul mot existant pour 'la pince à linge'), un chiné 'un enfant' (de pichinet ?), lou chume 'le plongeon' (terme emprunté au ligurien), l'escumairola 'l'écumoire', la fifa 'le trac', un gindroulh / un chinfroulh 'une personne vêtue de hardes', un(a) frandaioun(a) 'gamin(e) dépenaillé(e)', un lètou 'un grande personne portant des vêtements longs, un grand dadais' très souvent 'une personne sans intérêt', un lùfou 'un goinfre', un manja-scluèpe 'une nèfle', la mimia, de sens inconnu, utilisé dans l'expression sèmbla que manja ${ }^{34}$ la mimia 'il/elle mange avec dégoût', lou pega-feta 'le pain d'épice', li rateta 'les gamins', ou encore una subiola 'une très grande faim' (possible piémontisme, à rapprocher de l'expression bien niçoise avé $l i^{35}$ budèu que sublon).

La richesse lexicale de la langue des niçardophones naturels dépasse d'ailleurs le cadre du seul lexème comme le prouvent ces quelques idiotismes, apparemment oubliés des recueils : anà à la balha ${ }^{36}$ 'aller prendre un bain de mer', sanglut, dóu coù̀r mi vèn, va en aqueu / aquela que mi vòu bèn ('le hoquet me vient du cœur, il va à celui / celle qui m'aime', genre d'"à tes souhaits" que l'on dit à soi-même après le hoquet), trènta-nòu trènta-dès mot-à-mot 'trente-neuf, trente-dix' qui signifie 'on n'est pas à ça près', ...e d'aqueu prèire qu'en pissant la fahì vèire 'et ce fameux prêtre dont on voyait le membre quand il pissait' signifiant '...un ange passe', li va dounà lou pignatoun de la couòla ${ }^{37}$ 'il va lui refiler le pot de colle' pour dire 'il va lui créer des difficultés / lui rendre la monnaie de sa pièce', mangerii un can me la rougna / un cavau m'ai ferre / un bò me li couòrna 'je mangerais un chien galeux / un cheval avec ses fers / un bœuf avec ses cornes' comprendre : 'j'ai grand faim'.

Certains mots, bien connus ceux-ci, possèdent dans leur usage courant davantage de possibilités d'emploi que dans le niçois "officiel". Entrent dans cette catégorie le babau (qui n'est pas seulement le 'croquemitaine' mais également un simple 'chien' ou une 'petite bestiole', un 'insecte') ou la paire que forment les frugna et les muanda réputées n'être que la 'taie d'oreiller' et le 'caleçon' mais désignant l'un par rapport à l'autre respectivement le 'linge des femmes' et le 'linge des hommes'.

\subsection{Une accoutumance à la variation}

Le parler vulgaire est particulièrement sujet à la variation libre. Des unités lexicales aujourd'hui stabilisées dans nos publications ou nos salles de classe, sont diverses dans la langue populaire. Luèrna 'luciole', estraboutà 'dire n'importe quoi' ou 'sortir du sujet', boulun 'méli-mélo', repepilhà 'rabâcher', sànsoula 'pelle à grains' deviennent éventuellement livèrna ${ }^{39}$, estraboucà (ce verbe gagne avec sa mutation le sens de 'déformer (une langue)' et le caractère transitif : eu parla lou nissart ma l'estraboca 'lui, il parle le niçois mais il l'écorche'), boutun, rababilhà (l'agent est soit un repepiaire soit un rababeu), et sàrsoula (qui gagne également le sens d' 'objet sans valeur'). À vrai dire les polymorphes sont innombrables : fugairoun / fugairòu 'cuisinière' (appareil de cuisson), catigoulou / catigou / catigouòl 'chatouillis', chut(e) / chùtou 'chut', coumprà / croumpà 'acheter', mercat / marcat 'marché', ambouric ${ }^{40}$ / ambourigou 'nombril', còdou / gòdou 'galet', campié / gambié 'garde (champêtre), gendarme', plesi / plasi $^{41}$ ), etc.

Saisissons l'occasion de signaler des cas fréquents d'aphérèse et d'apocope qui sont bien logiquement rares ou absents dans une pratique châtiée : estai chut 'tais-toi', toutai doui 'tous les deux', espèça d'un ae 'espèce d'imbécile' deviennent généralement tai chut, tai doui, pèça d'un ae. A toutara 'à tout à l'heure', encara 'encore', pas encara 'pas encore' deviennent a toutà, encà, pancà.

De même constate-t-on un écart entre les paradigmes verbaux de référence et les usages. L'imparfait de l'indicatif du premier groupe à la personne 4 fait partie des exemples parlants. Il est réputé être de type cantavan. Or on peut entendre cantavavan, cantahavan, cantavian dans la vieille ville et cantian, cantièn dès les premières collines et dans tout le Moyen-Pays. Dans le même ordre d'idée, parallèlement à la forme propre du subjonctif présent à la personne 3 pour les verbes du premier groupe (vouòli que parle), la pratique urbaine manifeste une volonté de renoncement à marquer ce mode (vouòli que parla 'je veux 
qu'il parle'). D'autres hésitations sont également remarquables comme celle du verbe calé 'falloir', parfois réinterprété coulé, sans doute à cause du [o] de la prononciation moderne $\left[\mathrm{k}^{\prime} \mathrm{o}(\mathrm{w})\right]$ du présent de l'indicatif (cau 'il faut', cauria 'il faudrait', calia 'il fallait' aboutissent respectivement à còu, couria, coulia). Ce genre de phénomène est assez courant.

\subsection{Une obédience française implicite}

Dans un processus diglossique devenu automatique pour la dernière génération de locuteurs, le niçois de la rue comble ses lacunes et résout des déséquilibres en s'alignant sur la langue nationale. Aussi est-il truffé de francismes ${ }^{42}$, plus ou moins adaptés selon les cas à la phonétique et à la morphologie du dialecte; les cas de xénismes patents ne sont pas rares. La survenance de spécimens tels que lou bordereau (prononcé à la française), la pouagnada de la pouòrta 'la poignée de la porte', l'espouar 'l'espoir', voualà lou cadeau 'voilà le cadeau', faire un parì 'faire un pari', siéu touplen crouaiènt 'je suis très croyant ${ }^{43}$ est révélatrice de ce fait. Toutefois, s'ils menacent d'envahir le dialecte, ces emprunts sont pour les natifs, au même titre que n'importe quelle autre habitude sociolectique, des marqueurs de niçardité. Éventuellement, on se réclamera explicitement de cet usage. Durant l'entre-deux guerres déjà, Grinda ([1930 ?]) s'ouvre sur cette interrogation en guise d'avertissement : « aï scriché lou Nissarte que si parla anchieû c'est-à-dire... francisaté $?^{44} \gg$. L'évitement de certains francismes (et donc le purisme académique) peut même être interprété comme une mauvaise connaissance de la pratique des "vrais" locuteurs, sanctionnée par l'exclusion du groupe social ${ }^{45}$. Il est bien évident qu'il s'agit là d'un cas de subordination intouchable (Lamuela 1994: 147-149) que Sumien (2006: 48) résume ainsi : « une survalorisation des traits qui caractérisent un idiome : cela va jusqu'à la survalorisation des interférences des langues dominantes que l'on considère comme un emblème essentiel ».

\section{Le niçois académique : une progression funambulesque vers la forme juste}

\subsection{La sacralisation de l'écrit}

En réaction à une évolution débridée, une variation tentaculaire et une invasion étrangère massive, l'académisme a développé le goût de la forme juste. Si les plus félibréens de nos mainteneurs se réfugiaient volontiers dans le fonds occitan partagé ${ }^{46}$, désormais la tentation est plutôt d'affirmer sa nonprovençalité et de s'extirper par ses propres moyens des situations problématiques. Dès lors, le niçois s'élabore de façon autonome et les ouvrages linguistiques typiquement niçois deviennent la référence absolue, en particulier pour le courant académique majoritaire. Le courant occitaniste est lui obligé de louvoyer entre adhésion au modèle central et fidélité aux attestations parfois fantaisistes des dictionnaires niçois.

Car fantaisie il y a. Effectuant un relevé au hasard des attestations, sans principes fondamentaux, sans traitement de l'information et sans réflexion préalable sur le corpus, les dictionnaires sont précieux mais piégeux. Le principal outil lui-même ${ }^{47}$ est un mélange hétéroclite. Il compile, sans toujours les marquer, des usages relevant d'influences différentes (ambigù 'ambigu' < AMBIGUUS mais individuou 'individu' $<$ INDIVIDUUS, et non ambigù / individù ou ambiguou / individuou), d'époques différentes (on trouve des archaïsmes tels que quadrat mentionné à côté de carrat 'un carré') et d'espaces différents (présence de mots non occitans tels que margueria 'vacherie', recueilli dans la proche vallée ligurienne de la Roya). Hormis les cas où l'on s'en affranchit pour des raisons idéologiques (fidélité à une école ou suspicion d'influence étrangère), ces ouvrages de référence sont suivis jusque dans leurs errements. Leur statut de documents écrits autochtones contribue à sacraliser leurs propositions, largement reprises par tous les initiés, même si elles sont incomprises du locuteur naturel. Ainsi le suranné gaudina (attesté chez Raymond Feraud vers 1300) devient-il un moyen courant de désigner la forêt, à la place du simple bouòsc. Il ne faut pas voir dans ces exemples une intention normative (qui dépassait largement l'intention des auteurs) mais juste la volonté de partager des données rares. D’autres imprécisions sont regrettables. 
Souguigne par exemple, n'est pas vraiment un sourire, mais parce qu'il est donné comme équivalent du français sourire, il est partout employé dans ce sens, envoyant sourrire, riseta ou d'autres termes aux oubliettes ${ }^{48}$. Une simple traduction un peu approximative dans le dictionnaire français-niçois (rictus aurait, semble-t-il, mieux fait l'affaire), est érigé en vérité édifiante, puisque le dictionnaire devient la seule source de connaissance pour des locuteurs dont la langue maternelle est désormais le français, langue par laquelle ils passent pour traduire en niçois.

Les réalisations de beu, veu, aqueu évoquées plus haut étaient déjà un indice de cette sacralisation de l'écrit. Les prononciations naturelles ([iw], [yw]) sauvaient surtout le trait "fermé". Avec [cw] les mainteneurs se réfugiaient dans une prononciation compatible avec le $e$ graphique.

\subsection{Un certain retard}

Qui dit "forme juste" dit inévitablement "nécessité de figer les choses". Pendant ce temps le parler évolue. La norme prend donc mécaniquement du retard sur la réalité des faits. Cette réalité a simplifié la série des pronoms personnels objets, aujourd'hui reconnaissables à leur [i] ( $m i, t i, s i, n i, v i, s i)$ quand un état plus conservateur ( $m i, t i, s i$, nen, vous, si) semble préféré à l'écrit et dans l'enseignement. De même les quartiers, se rapprochant du provençal, éliminent la désinence -u de masculin pluriel (li ome, aqueli ${ }^{49}$ pichoui, quauque tipe, d'autre) là où l'écrit s'en sert encore largement (lu ome 'les hommes', aquelu pichoui 'ces enfants', quauqu tipe 'quelques types', d'autru 'd'autres', et l'on pourrait ajouter chertu 'certains', le mot chertou 'certain' ayant d'ailleurs complètement disparu de la bouche des locuteurs natifs, au même titre que son Doppelgänger félibréen cert /certou).

Le souci de se démarquer du français et du provençal peut même faire régresser le niçois à un stade, certes peu lointain, où l'italianisme avait sa place dans le vocabulaire savant ${ }^{50}$. L'italien ne touche pas le vocabulaire populaire mais ses mots savants pullulent dans l'écrit du XIXème siècle. La rue a abandonné ces formes, contrairement à l'académisme qui continue d'utiliser, un peu à contre-courant, des termes tels que nechessità, chivilisacioun, amichicia, counchepì, chircoundà que l'on cueille en feuilletant nos revues. Ces pratiques italianisantes chez les mainteneurs constituent une subordination intouchable en tout point semblable aux pratiques francisantes des locuteurs primaires. Admettons que cette accoutumance à l'italianité puisse jouer un rôle dans l'emploi spectaculaire de certaines formes proparoxytoniques, italianisantes ou non (scientificou, un simboulou, lou ritoumou, etc.) mais en tout cas oubliées du pavé. A vrai dire la tendance naturelle du niçois est, comme pour tous les parlers d'oc, à la suppression des proparoxytons ${ }^{51}$. D'anciens bouchers du Vieux-Nice nous ont affirmé qu'ils ne connaissaient pas d'autre mot que pelandra (pelàntrega, pelàndrega pour les lexicographes ${ }^{52}$ ) pour désigner l'aponévrose. Amenda 'amende' supplante généralement améndoula, làmpea 'lampe' est devenu lampa. La seconde guerre est jugée responsable de l'accélération d'un tel désamour pour les sdruccioli, mais tout porte à croire que le mouvement était déjà bien amorcé avant le conflit mondial ${ }^{53}$.

\subsection{Le risque de l'incohérence}

En effectuant des choix intuitifs au cas par cas, on prend le risque de créer une incohérence formelle entre plusieurs lexèmes équivalents. Devant $\left.{ }^{[}\right]$] et [j], la langue populaire réalise /e/ soit [i] soit [ $\varepsilon$ ]. Voilà pourquoi tous les lexèmes du genre de cenque 'ce que', lenga 'langue', arenga 'harangue' ou manelha

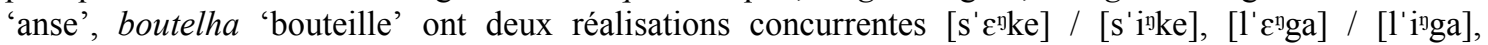

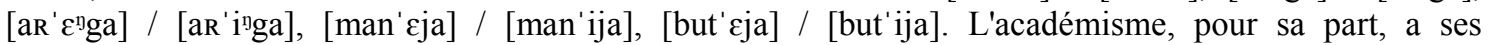
préférences. Elles découlent de choix par élimination. Si la variation libre de cenque ou manelha ne pose apparemment aucun problème, [l'inga] et [but'cja] semblent évités. Sans doute le premier est-il jugé à tort comme un italianisme flagrant. Dès lors on comprend mal la préférence pour aringa face à arènga ('harangue'). C'est un réflexe de même ordre qui pousse à fuir [but'cja], ressenti français, au profit de [but'ija]. L'influence extérieure n'a pourtant rien à voir dans ce phénomène. On est en présence d'un cas typique de distanciation maximale. Des choix, même motivés, brouillent donc des séries régulières, parfois de même type étymologique (manèlha $<$ MANICULA, boutilha $<$ BUTTICULA). Nous touchons déjà là à la question de l'hypercorrection. 


\subsection{L'écueil de l'hypercorrection}

Toujours le souci de conserver une distance maximale avec le français pousse à trouver toutes sortes d'expédients. Pour 'sourcil', le mot sourci du dictionnaire n'étant pas satisfaisant (il ressemble au français), et ussa fleurant trop la Provence, on trouve le nouveau-venu soubre-parpèla. L'invention, qui correspond à tout point de vue au génie de notre dialecte et témoigne de la compétence de son auteur, naît d'un effort qui n'était pas nécessaire. Sourcì n'a rien d'un emprunt récent mal prononcé. Il est vrai qu'ainsi orthographié on ne reconnaît pas notre $\operatorname{sourcilh}^{54}$, attesté un peu partout en Occitanie. Nos lexicographes ont fait ce qu'ils ont pu en consignant les formes qu'ils rencontraient. Or la prononciation des mots finissant par /-ij/, dont la désinence est tantôt tronquée (bisbilh 'chuchotement' est également attesté en tant que busbi), tantôt renforcée d'un appendice vocalique (boumbilh 'vrombissement' attesté sous la forme boumbilhi), génère systématiquement des hésitations.

La question de l'infinitif du verbe niçois signifiant 'voir' est également digne d'intérêt. Ce verbe se dit de deux manières dans la langue populaire, vèire et vé, le deuxième étant très courant. Comment se fait-il que seul vèire ait droit de cité dans la langue de référence ? Sans doute vé est-il considéré seulement comme une confusion ou une forme barbare de vèire ayant subi l'apocope. Il s'agit là d'un jugement assez hâtif et l'on peut trouver regrettable qu'un fait largement observé soit éliminé sans procès. 'Faire' ne se dit-il pas lui aussi soi faire soit fà, les deux possibilités étant parfaitement admises dans la langue littéraire?

La recherche de correction pourrait donc refuser des usages établis et conduire au discrédit d'unités authentiques. C'est dire si la complexité du fait linguistique exige une circonspection extrême de la part de quiconque ayant des velléités purificatrices.

\section{Conclusion}

Bien considéré mais mal entretenu, confronté à une problématique diglossique complexifiée par des représentations propres dues à son passé original, le niçois est menacé. Sa transmission repose sur les épaules de locuteurs secondaires dont le particularisme, mâtiné à divers degrés de classicisme, est rarement démenti. Pourtant, tributaires des outils qui leur permettent de traduire en niçois à partir du français, leur langue est assez éloignée de celle des natifs. C'est que les principaux dictionnaires, travaux d'amateurs passionnés, étaient destinés à des Niçois de langue maternelle occitane. Ils n'avaient pas vocation à servir de base à un standard. Mêlant anarchiquement divers marqueurs de nationalité, diverses variétés locales et diverses époques, ils ne font plus l'affaire pour des mainteneurs néolocuteurs. Sumien (2006 : 41) a identifié ce problème : «les occitanistes des nouvelles générations sont de plus en plus coupés de la langue héritée. Sans dictionnaires et sans grammaires complets, ils n'ont plus les moyens de cultiver un parler local. Sauzet [(1990:35)] explique qu'ils ne peuvent plus se contenter du do it yourself [...], il leur faut désormais du ready made $\gg$.

Ce ready-made, s'il a besoin d'une cohérence formelle (traitement régulier de la variation, graphie uniformisée, généralisation des cultismes non subordonnés, xénismes évités,...), ne doit pas non plus être une production à ce point théorique qu'elle oublie d'interroger la langue telle qu'elle se donne à nous. Aucune langue, aussi moderne ou normée soit-elle, ne peut se contenter d'un vocabulaire abstrait et de terminologies. Dans cette optique, le matériau didactique sera utilement complété par le parler des vieux quartiers, indispensable pourvoyeur d'unités lexicales. Les réalisations les plus surprenantes, sans être référentielles, pourront être mentionnées dans un but de documentation sur la réalité linguistique.

Ce n'est qu'à ces conditions que des ouvrages honnêtes, actuels et complets dans leur description pourront voir le jour. Alors, et dans l'hypothèse de la mise en place d'un plan institutionnel ambitieux, une véritable transmission sera possible et le niçois sera armé pour l'avenir. Nos locuteurs natifs ne sont pas éternels. Avec eux passera l'étalon de l'authenticité dialectale, à moins que la lexicographie niçoise ne se dote rapidement d'un usuel acceptant de prendre largement en compte leur enseignement. 


\section{Références bibliographiques}

Aracil, L. (1965). Conflit linguistique et normalisation linguistique dans l'Europe nouvelle. Nancy: Centre Universitaire Européen.

Bernissan, F. (2012). Combien de locuteurs compte l'occitan en 2012 ?, Revue de Linguistique Romane, 303-304, 467-512.

Carles, P. (inédit). Piccolo vocabolario nizzardo-italiano, 1866.

Castellana, G. (1947). Dictionnaire français-niçois. Nice : Éditions ludographiques françaises.

Castellana, G. (1949). Dictionnaire niçois-français. Nice : chez l'auteur.

Compan, A. (1954). Les Euvres de Rancher, La Nemaïda, La Mouostra raubada, Lou Fablié nissart. Publication spéciale de la Revue des Langues Romanes. Nîmes : Barnier.

Compan, A. (1967). Glossaire raisonné de la langue niçoise. Nice : Tiranty.

Dalbera, J.-Ph. (1983-1984). De la Genèse des proparoxytons dans les parlers nissarts, Travaux du cercle linguistique de Nice, 5-6, 33-62.

Dalbera, J.-Ph. (1988-1989). Phénomènes de contact, conflit de normes et changement linguistique. Le parler nissart urbain, Travaux du cercle linguistique de Nice, 10-11, 27-51.

Dalbera, J.-Ph. (1989). Interférences entre provençal et ligurien dans la genèse du système morphologique mentonnais, Bulletin du Centre de Romanistique et de Latinité tardive, 4-5, 89-97.

Dalbera, J.-Ph. (1994). Les Parlers des Alpes-Maritimes. Étude comparative. Essai de reconstruction. Londres : A.I.E.O.

Domenge, J.-L. (à paraître). Dictionnaire des parlers de la Tinée.

Eynaudi, J. (2009). Dictionnaire de la langue niçoise (édition intégrale). Nice : Acadèmia nissarda.

Fenouille, A. \& Rondelly, M. (1919). Li Beluga nissardi. Nice : Frey \& Trincheri.

Ferguson, C.A. (1959). Diglossia, Word, 15, 325-340.

Fishman, J. (1967). Bilingualism with and without diglossia, diglossia with and without bilingualism, Journal of social issues, XXIII, 2, 29-38.

Forner, W. (2001). Le Mentonnais entre toutes les chaises? Regards comparatifs sur quelques mécanismes morphologiques, Lexique Français-Mentonnais, 11-23. J.-L. Caserio \& Commission du vocabulaire mentonnais (éds.). Menton : Société d'Art et d'Histoire du Mentonnais.

Gardy, P. \& Lafont, R. (1981). La diglossie comme conflit : l'exemple occitan. Langages, 61, 75-91.

Gasiglia, R. (1982). Éloge de la vulgarité, Lou Sourgentin, 50, 50-54.

Gasiglia, R. (1984). Grammaire du Nissart, Nice : I.E.N.

Gasiglia, R. (2005). Comment les auteurs niçois ont représenté leur dialecte, Récits d'Occitanie, 143-169. Aix : Publications de l'Université de Provence.

Gelu, V. (1986), Euvres complètes. Raphèle-lès-Arles : CPM Marcel Petit.

Giordan, J. (1963). Lexique complémentaire du parler de la ville de Nice et des parlers environnants. Nice: Vincentelli.

Grinda, B. ([1930 ?]). Un Pessuqué de menchounada. Nice : chez l'auteur.

Isnard, P. (1949). Calendrier perpétuel. Nice : Éditions ludographiques.

Kremnitz, G. (1980). Démarche et particularités de la sociolinguistique catalane, Sociolinguistique: Approches, théories, pratiques, 21-33. Presses Universitaires de France. 
Kremnitz, G. (2002a). La Langue au miroir de l'histoire, Petite histoire sociale de la langue occitane, 13-44. Canet : Trabucaire.

Kremnitz, G. (2002b). Une Approche sociolinguistique, Petite histoire sociale de la langue occitane, 103-136. Canet : Trabucaire.

Lafont, R. (1971). Un problème de culpabilité sociologique : la diglossie franco-occitane, Langue française, 9, 93-99.

Lamuela, X. (1994). Estandardització i establiment de les llengües. Barcelone : Edicions 62.

Micèu, G. (1840). Grammatica Nissarda per emparà en pòou de temp lo patouas dòou paìs. Nice: Société typographique.

Nicola, J. (1972). L’Embourigou, Lou Pais Nissart, 3, 29-30.

Ninyoles, R.L. (1969). Conflicte lingüístic valencià. Valencia : Tres i Quatre.

Rondelly, M. ([?]). Li mieu douze Nova. Nice : Frey \& Trincheri.

Sauzet, P. (1990). La Grafia es mai que la grafia, Amiras, 21, 35-46.

Sumien, D. (2006). La Standardisation pluricentrique de l'occitan. Turnhout : Brepols.

Toselli, J.-B. (1864). Rapport d'une conversation sur le dialecte niçois. Nice : Cauvin.

Toselli, J.-B. (1878). Recuei de 3.176 prouverbi, sentensa, massima, conseu, parabola, buoi mot, precet \& diç nissart. Nice : Cauvin.

${ }^{1}$ Voir notamment Dalbera (1994) et Gasiglia (1984: 59-75).

${ }^{2}$ Bernissan (2012 : 469) cite dans le compte-rendu de sa propre enquête (la plus récente et la plus méthodique), celles « réalisées à la demande des Conseils Régionaux du Languedoc-Roussillon (1991), d'Aquitaine (1997, puis plus récemment en 2008), de Midi-Pyrénées (2010), de certains départements comme ce fut le cas dans les PyrénéesAtlantiques en 1994, dans les Hautes-Pyrénées en 1995, ou plus rarement à l'initiative des associations ». Ces travaux que nous avons consultés n'intéressent pas directement notre propos.

${ }^{3}$ Nous traduisons de l'original : « [the] stable maintenance rather than the displacement of one [code] by the other over time $»$.

${ }^{4}$ Voir Gardy \& Lafont (1981 : 78-82) et Kremnitz (2002a).

${ }^{5}$ Cité par Gardy \& Lafont (1981: 79).

${ }^{6}$ Lafont (1971 : 98) consacre un paragraphe aux particularités sociolinguistiques niçoises.

${ }^{7}$ «L'influence française a pénétré tardivement dans le Comté de Nice. [...] La domination du français s'est accrue en niçois depuis le XIXème siècle mais elle reste moins profonde que dans la plupart des parlers occitans » (Sumien $2006: 31)$

${ }^{8}$ Voir Gelu $(1986: 23)$

${ }^{9}$ Préface de "La Mouostra raubada" in Compan (1954). Nous respectons la graphie française originale de l'auteur.

${ }^{10}$ L'absence d'enquête est regrettable pour ce fait évident qui eût été facilement vérifiable : «La ville de Nice est un cas particulier. Bien qu'elle soit au bout de l'axe de circulation Paris-Rhône-Basse-Provence, elle semble mieux résister à la substitution linguistique que les autres grandes métropoles occitanes. On dit souvent que les Niçois nés avant la Seconde Guerre Mondiale sont occitanophones, mais aucune enquête ne le démontre. Cela pourrait s'expliquer par l'intégration tardive à la France et le fort particularisme niçois ». (Sumien $2006: 32$ )

${ }^{11}$ Gasiglia (1984: 73) présente ce phénomène dans ses grandes lignes. Dalbera (1988-1989: 30 et $\left.1987: 52\right)$ évoque également l'extension géographique de certains traits qui correspondent à ceux du niçois. Nous avons nous-même constaté que certaines particularités locales recueillies il y a encore trente ans ont disparues au profit de l'usage niçois. Les locuteurs naturels de Levens ont, par exemple, désormais abandonné leur article défini masculin singulier sou pour lou. 
${ }^{12}$ Un chercheur de l'Université de Nice nous confiait, il y a peu, avoir dû recommencer l'enquête dialectologique qu'il effectuait dans un village : dans un premier temps son informateur avait répondu à toutes les questions en niçois urbain plutôt que dans sa variété locale.

${ }^{13}$ D'après Kremnitz (2002a : 28-29), au cours des deux derniers siècles « les gens se [sont] regroup[és] [...] sur les bords de la Méditerranée », le département des Alpes-Maritimes passant selon ses chiffres de 199.000 habitants en 1872 à 815.000 habitants en 1975. On dépasse aujourd'hui le million d'habitants.

${ }^{14}$ Sur cette question, voir Gasiglia (2005).

${ }^{15}$ Nous entendons par là l'état de langue actuel qui s'est dégagé au tournant du XIXème siècle pour ne connaître que des évolutions de détail jusqu'à ce jour.

${ }^{16}$ Gasiglia (1982). Voir également Dalbera (1988-1989).

${ }^{17}$ C'est bien de cela qu'il s'agit, même si ce mot n'était pas alors utilisé par les mainteneurs du niçois.

${ }^{18}$ Publiée à raison d'un numéro par an (à deux exceptions près) de 1929 à 1941 par la Soucietà felibrenca dóu Cairèu, héritière de l'Escola de Bellanda. Y collaboreront des grands noms de la littérature niçarde comme Louis Genari et Francis Gag.

${ }^{19}$ Elle intègre à l'occasion certains de ses usages. On trouve chez les auteurs d'obédience félibréenne des termes tels que uèch (pour vuèch) ce que (pour cen que), barrulà (pour barrounà), frairal (préféré aux plus courants fratern Ifratèrnou et fraternel (francisme), 'fraternel'), gramaci 'merci', urousa pour uroua, ou des tics de la prose provençale comme les basta! qui servent à changer de sujet à la manière d'un bref français.

${ }^{20}$ Ils sont tous deux soucieux d'une grande fidélité au latin. On trouve dans Isnard (1949) espunimen 'exposition', gubernament 'gouvernement', protetris 'protectrice', l'armada imperial 'l'armée impériale' ainsi qu'une foule de propositions du même genre. Elles contrastent avec les plus habituels espousicioun, gouvern / gouvernou, proutetriça, armada imperiala jugés déviants.

${ }^{21}$ Voir Kremnitz (2002b : 119) « la compétence linguistique [des locuteurs secondaires] n'atteint que dans de très rares cas le niveau de celle des locuteurs primaires ». La situation décrite plus en détail par Bernissan (2012 : 475476) à propos des Hautes-Pyrénées correspond également à ce que l'on constate à Nice «Les compétences linguistiques [des néolocuteurs] sont correctes, voire très correctes ». Mais « les règles régissant la concordance des temps ne sont pas toujours appliquées. La réalisation de certains phonèmes est parfois éloignée de la prononciation des locuteurs naturels (le degré d'aperture des voyelles par exemple). Il peut arriver que le lexique utilisé soit déterritorialisé. [...] Rappelons que cette catégorie (majoritairement des professionnels de la langue) est la seule à transmettre la langue occitane. La langue qu'ils transmettent n'est déjà plus la langue que parlent les locuteurs natifs ».

${ }^{22}$ Respectivement: 'ce n'est pas grave' pour fa pas rèn, 'une très belle maison' pour, à la rigueur, una maion d'aqueli bèli, 'le dix-septième siècle' à côté de lou XVIIème siècle / sècoulou, 'particulier' en face de particulié, 'contemporain' à côté de countempouran, 'septième' à côté de setième, 'tatillon' qui vient s'ajouter à pachoun, patet.

${ }^{23}$ Pour les questions de standards régionaux d'un occitan normé, voir Sumien (2006 : 151-176) et en particulier §IX.9.3 "Provençal niçois : une personnalité affirmée" où l'auteur écrit au sujet de ce dialecte que «la codification reste fragile $»($ p. 168).

${ }^{24}$ On le trouve par exemple dans estaís 'rassis', levaís 'amovible, meuble', cantaissa 'chanson interminable'.

${ }^{25}$ Les données orales du parler de la rue ont été récoltées auprès d'habitants du Vieux-Nice que nous avons enregistrés. Ces informateurs ont ou auraient, s'ils étaient encore en vie, entre 75 et 95 ans.

${ }^{26}$ Dalbera (1994 : 348-349).

${ }^{27}$ Dans Dalbera (1994) c'est néanmoins la transcription phonétique [o] que l'on trouve pour cette voyelle. 
${ }^{28}$ Le rôle du ié félibréen doit peser dans la balance. L'occitanisme attribue également d'emblée (à tort selon nous) la prononciation [je] à toute la Provence, à laquelle il rattache Nice.

${ }^{29}$ Dans ce cas la paire /bew/ 'il boit' /bew/ 'beau' est malmenée.

${ }^{30}$ Gasiglia (1984 : 26)

${ }^{31}$ Sur l'origine de la diphtongue dans ce mot, tout incite à penser que la semi-consonne n'est pas le résultat du L de COLPUS mais ce qu'il reste d'un ancien [p] (còp), comme dans tròu (tròp) 'trop', sau (sap) 'il sait', un cau (un cap) 'un cap'. De ce fait, la vogue qui consiste à noter ce mot còup mériterait d'être discutée.

${ }^{32}$ Par ex. [sjew an'a] pour [sjew an'at ${ }^{\mathrm{e}}$ 'je suis allé'.

${ }^{33}$ Pour tu que la counouisses 'toi qui la connais'.

${ }^{34}$ Mange, dans la langue soignée.

${ }^{35} \mathrm{Lu}$, dans la langue soignée.

${ }^{36}$ Expression que l'on rencontre en français populaire : aller à la baille.

${ }^{37}$ Pour pega, 'poix, colle'.

${ }^{38}$ Pour bana, 'corne'.

${ }^{39} \mathrm{Ce}$ mot explique peut-être une variante de la chanson Es lou festin dei verna, que tant bèn nen gouverna qui devient (via un festin de li verna ?) Au festin dei liverna si canta li lanterna.

${ }^{40}$ Nous tombons par hasard sur embouric et embourigou dans Nicola (1972:29).

${ }^{41}$ Il est assez surprenant que plesì l'emporte si largement et depuis longtemps à l'écrit. Les locuteurs utilisant plasì ne sont pourtant pas si rares.

${ }^{42}$ Nous préférons ce mot à gallicisme, la langue d'oc étant apparue sur des territoires gaulois.

${ }^{43}$ Pour lou bourdarèu, la pougnada, l'espèr, aquì / vaquì / vequì lou regal, faire un'escoumessa, siéu touplen credènt (la tournure n'est pas très niçoise). L'évitement de termes proches de l'italien semble évident (regalo, scommessa, credente)

${ }^{44}$ « J'ai écrit le niçois qui se parle de nos jours, c'est-à-dire... francisé ?».

${ }^{45}$ Confrontés au niçois académique, nos informateurs peuvent avoir des réactions du genre de "Où vont-ils pêcher ces mots ?" "On ne dit pas ça comme ça". On constate chez certains une véritable défiance vis-à-vis des enseignants : "ils ne parlent pas comme nous", avec une part de fantasme : "ils ne sont pas d'ici". Un ami du groupe d'informateurs que nous fréquentons régulièrement nous a même lancé un jour le surréaliste : "Tu es prof de niçois ? Ah bon, mais tu le parles bien", où bien n'est vraisemblablement pas - vu notre niveau en tant que locuteur - à interpréter comme un jugement de valeur mais plutôt comme la validation du code utilisé.

${ }^{46}$ Giordan $(1963$ : 149, 166) profite d'attestations anciennes pour réintroduire des unités disparues à Nice mais largement utilisées par les félibres de Provence telles que rènc (XVème siècle) 'rang' et sorn (XIIIème siècle) 'sombre'. Compan (1967 : 107) impose d'autorité les standards provençaux comme ussa 'sourcil'. Il signale d'autre part un étrange lu belori (à côté de l'incontestable li belúrias 'les atours'). Il s'agit en fait du nom féminin provençal belòria, réalisé [bel'ori] dans un grande partie de la Provence, et donc souvent orthographié belòri ce qui lui a fait croire qu'il s'agissait d'un masculin. L'introduction arbitraire et non concertée de ce mot dans un ouvrage niçois s'est donc mal passée...

${ }^{47}$ Castellana (1947 et 1949). 
${ }^{48}$ Cet exemple nous permet de souligner le danger auquel on s'expose en n'ayant que des outils bilingues et aucun usuel monolingue où le dialecte soit autonome.

${ }^{49}$ Qui reste pour l'instant en concurrence avec aquelu [ak'œlœ]

${ }^{50}$ Quelques traces en subsistent, notamment dans l'arrière pays, comme mèdicou 'médecin', sanitatèvoul 'sain' ou fede 'foi' dans la Tinée. Cf. Domenge (à paraître).

${ }^{51}$ Ce qui a déjà été remarqué. Dalbera (1983-1984 : 56).

${ }^{52}$ Cf. Eynaudi (2009 : 905), Giordan (1963 : 87) et Toselli (1864:217).

${ }^{53}$ Beaucoup d'entre eux semblent invraisemblables à nos informateurs, nés dans les années 1920 ou 1930. Déjà chez Rondelly ([?] : 5) feriga remplace ferìgoula. L'auteur est né en 1854 et décédé en 1935.

${ }^{54}$ On le reconnaît mieux dans Carles (inédit) où il est graphié sorsii. Les troubadours utilisaient sobrecilh. 